\title{
Improving care for people with diabetes
}

\section{Social inequality and access to care}

The purpose of the National Service Framework for Diabetes is to establish national standards for the care of people with diabetes to improve care for all and to reduce inequalities. The harmful effect of social deprivation on health is well recognised ${ }^{1}$ and is mirrored in diabetes where "wealthy means healthy". In patients with diabetes increased social deprivation has been shown to be related to higher hospital admission rates, ${ }^{3}$ poorer diabetes control, increased complications, and increased mortality. ${ }^{4}$ However, the reasons for these inequalities and the relative contributions of differences in individual behaviour and access to high quality health care are unclear. Goyder and colleagues $^{5}$ in this issue of Quality in Health Care have elucidated how social factors might influence access to diabetes care. In a cohort of individuals with diabetes identified from general practice, they examined which factors predict attendance for diabetes review at both hospital clinics and general practices. The predictors of hospital attendance were younger age, longer duration of diabetes and treatment with insulin, access to a car, home ownership, and non-manual occupation; living in a deprived area was not a predictor. In contrast, the predictors of attendance for review in general practice were older age, less co-morbidity, and being white. Though living in a more deprived area was related to a reduced chance of review in general practice, individual socioeconomic indicators were not. The authors suggest that inequality at the general practice level may be due to practices in more deprived areas being less likely to offer well organised diabetes care. If good quality care is available locally, individual socioeconomic factors are less likely to be important. This study shows that small area information can hide inequalities at the individual level, and that the factors influencing access to care are complex.

\section{The "missing" annual reviews}

While there is no clear consensus as to where treatment of the diabetic patient should be provided, there is widespread agreement that, following initial assessment soon after diagnosis, care should take the form of a regular structured review every 1-2 years (the annual review) with interim follow up as necessary. ${ }^{6}$

Evidence-based national clinical practice guidelines for the management of type 2 diabetes are being developed. The paper by Melville et $a l^{7}$ in this issue is based on systematic reviews of the two most common microvascular complications of the disease-diabetic retinopathy and foot problems. They found that systematic review of trial evidence for retinopathy showed that screening is highly effective in preventing blindness when combined with laser treatment. They recommend that screening should be provided for all people with diabetes; the service should be organised locally to ensure adequate population coverage and should be provided by accredited optometrists or by mobile retinal photography units operating in a variety of locations. Direct ophthalmoscopy by general practitioners is not recommended. Consensus amongst expert groups is that annual screening is appropriate.

Fifteen percent of people with diabetes develop foot ulcers. The ulcers are highly susceptible to infection leading to massive tissue destruction and possible amputation.
The recurrence rate is high $(70 \%$ in five years). Melville et $a l^{7}$ found that multidisciplinary interventions such as education to increase patients' knowledge about foot care, podiatry, and therapeutic shoes can improve the condition of the feet and help to reduce ulcer and amputation rates. Various treatments are used for diabetic foot ulcers but the evidence for their effectiveness is poor. In this condition prevention seems to be more promising than cure, but is dependent on first identifying the "at risk" foot and then providing appropriate interventions. Deformities, previous ulceration, neuropathy, or ischaemia make the diabetic foot "at risk". These can all be detected by simple non-invasive screening tests at annual structured review.

The UK Audit Commission's recent national survey of diabetes services ${ }^{8}$ discovered that two thirds of hospital sites could not tell how many people had had structured reviews in the previous 18 months. This was attributed to poor record keeping rather than poor care, but even the hospitals which did keep this information estimated that only two thirds to three quarters of patients had had structured reviews. What is happening to the patients who are not having their annual review in hospital? Are they having annual reviews in general practice? Goyder et $a l^{5}$ defined a routine diabetes review in general practice as three out of seven diabetes related examinations being carried out on one occasion. They found that a third of diabetic patients had not had any such review in general practice, nor had they attended the hospital diabetes clinic during the five year period covered by the study. Their findings suggest that a proportion of patients are not having regular structured care anywhere. The UKPDS has shown that good control of blood pressure and glycaemia leads to a reduction in the rate of complications ${ }^{9}$ but achieving these targets will be very difficult if many patients are not having regular review. Primary and secondary care will need to work together to improve this situation, but cooperation across the primary/secondary interface is currently suboptimal. ${ }^{10}$ Tackling the issue of inadequate follow up presents a challenge for the implementation strategy of the National Service Framework. ${ }^{11}$

MARY PIERCE

Senior Lecturer in General Practice,

Imperial College School of Medicine,

London SW10 9NH, UK

1 Black D, Morris JN, Smith C, et al. Report of the working group on inequalities in health. London: Department of Health and Social Security, 1980.

2 Ward J. Wealthy means healthy: diabetes and social deprivation. Diabetic Med 1994;11:334-5.

3 Kelly WF, Mahmood R, Kelly MJ, et al. Influence of social deprivation on illness in diabetic patients. BMF 1993;307:115-6.

4 Robinson N, Lloyd CE, Stevens LK. Social deprivation and mortality in adults with diabetes mellitus. Diabetic Med 1998;15:205-12.

5 Goyder EC, McNally PG, Botha JL. Inequalities in access to diabetes care: evidence from a historical cohort study. Quality in Health Care 2000;9:859 .

6 Department of Health. Health service guidelines, 1997. Key features of a good diabetes service. HSG (97) 45 and Welsh Office WHC 98 (35). London: Department of Health, 1997

7 Melville A, Richardson R, McIntosh A, et al. Complications of diabetes: screening for retinopathy and management of foot ulcers. Quality in Health Care 2000;9:137-41

8 UK Audit Commission. Testing times: a review of diabetes services in England and Wales. London: UK Audit Commission, 2000.

9 Mogensen CE. Combined high blood pressure and glucose in type 2 diabetes: double jeopardy. BMF 1998;317:693.

10 Pierce M, Agarwal G, Ridout D. A survey of diabetes care in general practice in England and Wales. Br F Gen Pract 2000 (in press).

11 Department of Health. A first class service. Quality in the new NHS. London: Department of Health, 1998. 